\title{
Perturbations for Slotheon Field Dark Energy and its Evolution
}

\section{Debasish Majumdar*}

Saha Institute of Nuclear Physics, HBNI, 1/AF Bidhannagar, Kolkata 700064, India

E-mail: debasish.majumdaresaha.ac.in

\section{Upala Mukhopadhyay}

Saha Institute of Nuclear Physics, HBNI, 1/AF Bidhannagar, Kolkata 700064, India

E-mail: upala.mukhopadhyayesaha.ac.in

\begin{abstract}
We consider in this work a Slotheon scalar field for Dark Energy. A Slotheon field is inspired by extra dimensional models of Dvali, Gabadadze and Porrati (DGP) related to brane world. In the decoupling limit of this model, the DGP theory in Minkowski space-time is described by a scalar field that obeys a shift symmetry. When extended to curved space time this scalar field is termed as Slotheon field. In this work we calculate general relativistic perturbations by deriving the Dark Energy and matter perturbation equations and solve them numerically. The results are then compared with similar quantities derived from $\Lambda$ CDM model. It appears that the Slotheon field model for Dark Energy is more akin to Dark energy in $\Lambda$ CDM model than that in standard Quintessence model. A comparison has also been made between the matter power spectra obtained from Slotheon model and $\Lambda$ CDM model.
\end{abstract}

European Physical Society Conference on High Energy Physics - EPS-HEP2019 -

10-17 July, 2019

Ghent, Belgium

* Speaker. 


\section{Introduction}

The source of Dark Energy that constitutes about $68.5 \%$ of the total content of the Universe and is thought to have caused the late time acceleration of the Universe still remains an enigma. Although the cosmological constant $\Lambda$ is popularly used to interpret Dark Energy but it is associated with cosmic coincidence problem [1]. Also, if the cosmological constant is attributed to the vacuum energy then a fine tuning of the order of $10^{121}$ is required to be made. The other favourite interpretation of Dark Energy is the quintessence Dark Energy [2] where, unlike the cosmological constant the Dark Energy is considered to be varying with time and generally formulated by considering a scalar field $\phi$ with the potential $V(\phi)$ whereby the change of $\phi$ is very slow as time progresses.

In this work we also consider a scalar field driven varying Dark Energy that causes the late time acceleration of the Universe but in this case the origin of the scalar field is from an extra dimensional model. In particular we consider a scalar field arising out of the Dvali, Gabadadze and Porrati (DGP) [3] extra dimensional model that originates from the braneworld. The basic concept of this model is related to the understanding that the localised gravity in four dimensions (brane) weakens at large distances. As the Planck's mass $M_{\mathrm{pl}} \rightarrow \infty$ and when the crossover scale $r_{c}$ for transition from four dimensions to five dimensions also tends to infinity $\left(r_{c} \rightarrow \infty\right)$, the theory can be described by a scalar field $\pi[4,5]$ with a shift symmetry $\pi \rightarrow \pi+a+b_{\mu} x^{\mu}$, where $a$ and $b_{\mu}$ represent a constant and a constant vector respectively. The scalar field (in Minkowski space) which describes this theory in curved spacetime is termed as Slotheon scalar field. In this work the Dark Energy and their perturbations as well as the late time acceleration are addressed with this Slotheon field model.

The inhomogeneities in Dark Energy are expressed in terms of the Dark Energy perturbations. The perturbations are important to obtain the matter power spectrum. We calculate these perturbations as also their evolutions for the Slotheon Dark Energy models and obtain the power spectrum.

In section 2 we discuss the formalism of the background evolutions and the evolutions of perturbations for Slotheon field Dark Energy. In section 3 the results are discussed. Finally a summary is given in section 4 .

\section{Background Evolution and Perturbation of Slotheon Field Dark Energy}

The Lagrangian density of the Slotheon field $\pi$ is written in terms of the covariant derivative, $\pi_{; \mu}$ of $\pi$, the Einstein's tensor $G_{\mu \nu}$, the potential $V(\pi)$ and by adding the Einstein-Hilbert term. Note that this field $\pi$ moves slower than the ordinary scalar in scalar field theory and hence the nomenclature. The action for the Slotheon field is given as [6]

$$
S=\int d^{4} x \sqrt{-g}\left[\frac{1}{2}\left(M_{\mathrm{pl}}^{2} R-\left(g^{\mu \nu}-\frac{G^{\mu v}}{M^{2}}\right) \pi_{; \mu} \pi_{; v}\right)-V(\pi)\right]+S_{m}\left[\psi_{m} ; e^{2 \beta \pi / M_{\mathrm{pl}}} g_{\mu \nu}\right],
$$

where $\beta$ is a dimensionless coupling with matter field and field $\pi$. By varying the above action the equations of motion for the field $\pi$ are obtained as

$$
\begin{aligned}
M_{\mathrm{pl}}^{2} G_{\mu v} & =T_{\mu \nu}^{(m)}+T_{\mu v}^{(\pi)}, \\
\square \pi+\frac{1}{M^{2}}\left[\frac{R}{2} \square \pi-R^{\mu v} \pi_{; \mu \nu}\right]-V_{\pi} & =-\frac{\beta}{M_{\mathrm{pl}}} T^{(m)},
\end{aligned}
$$


where the radiation component of the energy momentum tensor $T_{\mu \nu}$ is not included. The above symbols above have their usual significance. The energy momentum tensor for field $\pi, T_{\mu \nu}(\pi)$ is given by

$$
\begin{aligned}
T_{\mu \nu}^{(\pi)}= & \pi_{; \mu} \pi_{; v}-\frac{1}{2} g_{\mu v}(\nabla \pi)^{2}-g_{\mu v} V(\pi)+\frac{1}{M^{2}}\left(\frac{1}{2} \pi_{; \mu} \pi_{; v} R-2 \pi_{; \alpha} \pi_{(; \mu} R_{v}^{\alpha}\right) \\
& +\frac{1}{2} \pi_{; \alpha} \pi^{; \alpha} G_{\mu v}-\pi^{; \alpha} \pi^{; \beta} R_{\mu \alpha v \beta}-\pi_{; \alpha \mu} \pi_{; v}^{\alpha}+\pi_{; \mu v} \pi_{; \alpha}^{\alpha} \\
& \left.+\frac{1}{2} g_{\mu v}\left(\pi_{; \alpha \beta} \pi^{; \alpha \beta}-\left(\pi_{; \alpha}^{\alpha}\right)^{2}+2 \pi_{; \alpha} \pi_{; \beta} R^{\alpha \beta}\right)\right) .
\end{aligned}
$$

In the above $R_{\mu \alpha v \beta}$ denotes a Riemann curvature tensor. For a spatially flat background, assuming the coupling $\beta=0$ the equations of motion are now written as

$$
\begin{gathered}
3 M_{\mathrm{pl}}^{2} H^{2}=\rho_{m}+\frac{\dot{\pi}^{2}}{2}+\frac{9 H^{2} \dot{\pi}^{2}}{2 M^{2}}+V(\pi), \\
M_{\mathrm{pl}}^{2}\left(2 \dot{H}+3 H^{2}\right)=-\frac{\dot{\pi}^{2}}{2}+V(\pi)+\left(2 \dot{H}+3 H^{2}\right) \frac{\dot{\pi}^{2}}{2 M^{2}}+\frac{2 H \dot{\pi} \ddot{\pi}}{M^{2}}, \\
0=\ddot{\pi}+3 H \dot{\pi}+\frac{3 H^{2}}{M^{2}}\left(\ddot{\pi}+3 H \dot{\pi}+\frac{2 \dot{H} \dot{\pi}}{H}\right)+V_{\pi} .
\end{gathered}
$$

The form of the potential $V(\pi)$ is taken as $V(\pi)=V_{0} \exp \left(-\frac{\lambda \pi}{M_{\mathrm{pl}}}\right)$ where $\lambda$ is a constant.

In order to compute the perturbations for the Slotheon field $\pi$ we first write the scalar perturbed metric in Newotonian gauge as [7]

$$
d s^{2}=-(1+2 \Phi) d t^{2}+a^{2}(t)(1+2 \Psi) \delta_{i j} d x^{i} d x^{j},
$$

where $\Phi$ and $\Psi$ are the gravitational potential and spatial curvature perturbation respectively and $a(t)$ is the scale factor. For no anisotropic stress $\Phi=-\Psi$ and considering the matter to be a perfect fluid we have $T_{v}^{\mu}=(\rho+p) u^{\mu} u_{v}+p \delta_{v}^{\mu}$. The quantities such as density, pressure, velocity and field etc. can be expressed as the sum of their respective unperturbed components and the perturbations as given in the following equations

$$
\begin{aligned}
\rho(t, \vec{x}) & =\bar{\rho}(t)+\delta \rho(t, \vec{x}), \\
p(t, \vec{x}) & =\bar{p}(t)+\delta p(t, \vec{x}), \\
u^{\mu} & =\bar{u}^{\mu}+v^{\mu} \\
\pi(t, \vec{x}) & =\bar{\pi}(t)+\delta \pi(t, \vec{x})
\end{aligned}
$$

and similarly the energy momentum tensor $T_{v}^{\mu}$ is expressed as $T_{v}^{\mu}=\bar{T}_{v}^{\mu}+\delta T_{v}^{\mu}$. For the Slotheon field tensor $T_{\mu \nu}^{\pi}$ the perturbations are obtained as

$$
\begin{gathered}
\delta \rho_{\pi}=-\frac{1}{a^{3} M^{2}}\left[-a^{3} M^{2} \delta \pi V_{\pi}+\dot{\pi}\left(2 \dot{a} \nabla^{2}(\delta \pi)-\delta \dot{\pi}\left(a^{3} M^{2}+9 a \dot{a}^{2}\right)\right.\right. \\
\left.\left.+\dot{\pi} a\left(a^{2} M^{2} \Phi-\nabla^{2} \Phi+9 \dot{a}(a \dot{\Phi}+2 \Phi \dot{a})\right)\right)\right], \\
\left(\bar{\rho}_{\pi}+\bar{p}_{\pi}\right) v_{i}=-\left.\frac{\dot{\pi}}{a^{2} M^{2}}\left[-2 a \delta \dot{\pi} \dot{a}+\delta \pi\left(a^{2} M^{2}+3 \dot{a}^{2}\right)+a(a \dot{\Phi}+3 \Phi \dot{a}) \dot{\pi}\right]\right|_{i},
\end{gathered}
$$




$$
\begin{aligned}
\delta p_{\pi}= & \frac{1}{a^{3} M^{2}}\left[-a^{3} M^{2} \delta \pi V_{\pi}+\dot{\pi}\left(-2 a^{2} \delta \ddot{\pi} \dot{a}+a\left(-a^{2} M^{2} \Phi+a^{2} \ddot{\Phi}+\nabla^{2} \Phi-\Phi_{i i}\right) \dot{\pi}\right.\right. \\
& +\dot{a}\left(\nabla^{2}(\delta \pi)-\delta \pi_{i i}+2 a(3 a \dot{\Phi}+\Phi \dot{a}) \dot{\pi}\right)+a \delta \dot{\pi}\left(-\dot{a}^{2}+a\left(a M^{2}-2 \ddot{a}\right)\right) \\
& \left.\left.+4 \Phi \dot{\pi} \ddot{a} a^{2}\right)+a\left(\nabla^{2} \delta \pi-\delta \pi_{i i}-2 a \delta \dot{\pi} \dot{a}+2 a(a \dot{\Phi}+4 \Phi \dot{a}) \dot{\pi}\right) \ddot{\pi}\right] .
\end{aligned}
$$

In order to obtain the equations of motion for perturbations, we need to solve the Einstein's equation $\delta G_{v}^{\mu}=8 \pi G \delta T_{v}^{\mu}$ for perturbed quantities. Working in the Fourier space (replacing $\nabla^{2}$ by $-k^{2}$ ) the perturbed equations of motion are written as [8]

$$
\begin{aligned}
3 H^{2} \Phi+3 H \dot{\Phi}+\frac{k^{2} \Phi}{a^{2}} & =-4 \pi G \sum_{i} \delta \rho_{i}, \\
k^{2}(\dot{\Phi}+H \Phi) & =4 \pi G a \sum_{i}\left(\bar{\rho}_{i}+\bar{p}_{i}\right) \theta_{i}, \\
\ddot{\Phi}+4 H \dot{\Phi}+2 \dot{H} \Phi+3 H^{2} \Phi & =4 \pi G \sum_{i} \delta p_{i} .
\end{aligned}
$$

Note that we have not changed the notations for $\Phi, \delta \rho_{i}, \delta p_{i}$ etc. for brevity. In the above, the summation is over matter perturbations and the Dark Energy perturbations due to Slotheon field and $k=\frac{2 \pi}{\lambda_{p}}$ where $\lambda_{p}$ is the length scale of the perturbation. Also the perturbation $\delta \pi$ of the Slotheon field $\pi$ is obtained as (using Eqs. 2.1, 2.8)

$$
\begin{gathered}
\frac{1}{M^{2}}\left[H \dot{\pi} \frac{k^{2} \Phi}{a}+18 \Phi \dot{a}^{3} \dot{\pi}-2 \ddot{a} k^{2} \delta \pi+2 \dot{a} \dot{\pi} k^{2} \Phi-3 \dot{a}^{3} \delta \dot{\pi}+H^{2} a\left(-k^{2} \delta \pi\right)+\right. \\
\frac{\dot{\pi} k^{2} \dot{\Phi}}{a}-a^{3} M^{2} V_{\pi} \delta \pi+2 M^{2} a^{3} \Phi\left(V_{\pi}+2 \ddot{\pi}\right)+4 a^{3} M^{2} \dot{\pi} \dot{\Phi}+\dot{\pi} a^{3} k^{2} \dot{\Phi}- \\
a^{3} M^{2} \delta \ddot{\pi}+36 a^{2} \Phi \dot{\pi} H \ddot{a}+18 \Phi a^{3} H^{2} \ddot{\pi}-M^{2} a k^{2} \delta \pi+2 \ddot{\pi} a k^{2} \Phi-6 a^{2} H \ddot{a} \delta \dot{\pi}+ \\
30 a^{3} H^{2} \dot{\pi} \dot{\Phi}+2 \dot{\pi} a k^{2} \dot{\Phi}-3 a^{3} H^{2} \delta \ddot{\pi}+12 a^{3} M^{2} \Phi H \dot{\pi}+6 \dot{\pi} a^{2} \ddot{a} \dot{\Phi}- \\
\left.a^{3} H\left(3 M^{2} \delta \dot{\pi}+6 \ddot{\pi} \dot{\Phi}-\dot{\pi} k^{2} \Phi-6 \dot{\pi} \ddot{\Phi}\right)\right]=0 .
\end{gathered}
$$

\section{Numerical Solutions of Perturbation Equations and Results}

The linearised perturbation equations (Eqs. 2.16 - 2.19) are now solved numerically along with the background equations (Eqs. 2.5 - 2.7) to obtain the evolutions of the cosmological density parameter $(\Omega)$, the Dark Energy equation of state $(\omega)$, Dark Energy density fluctuations $\delta_{\pi}\left(=\frac{\delta \rho_{\pi}}{\bar{\rho}_{\pi}}\right)$ and matter density fluctuations $\delta_{m}\left(=\frac{\delta \rho_{m}}{\bar{\rho}_{m}}\right)$. To this end certain dimensionless variables defined as $x=\frac{\dot{\pi}}{\sqrt{6} H M_{\mathrm{pl}}}, y=\frac{\sqrt{V(\pi)}}{\sqrt{3} H M_{\mathrm{pl}}}, \lambda=-M_{\mathrm{pl}} \frac{V_{\pi}}{V(\pi)}, \varepsilon=\frac{H^{2}}{2 M^{2}}, q=\delta \pi / \frac{d \pi}{d N}(N=\ln (a)$ is the number of e-foldings), will be useful. The equations are then written in terms of these dimensionless variables.

Thus we obtain the equation of state and density parameters as

$$
\begin{aligned}
\Omega_{\pi} & =y^{2}+x^{2}(1+18 \varepsilon), \\
\Omega_{m} & =1-y^{2}-x^{2}(1+18 \varepsilon), \\
\omega_{\mathrm{eff}} & =\frac{p_{\text {total }}}{\rho_{\mathrm{total}}}=-1-\frac{2 \dot{H}}{3 H^{2}} \\
\omega_{\pi} & =\frac{\omega_{\mathrm{eff}}}{\Omega_{\pi}} .
\end{aligned}
$$


In the above $\Omega=\frac{\bar{\rho}_{i}}{\rho_{c}}$ ( $i=\pi, m$ etc. and $\rho_{c}$ is the critical density of the Universe), $p_{\text {total }}=p_{m}+p_{\pi}$, $\rho_{\text {total }}=\rho_{m}+\rho_{\pi}$ and $\omega_{\pi}$ is the Slotheon field Dark Energy equation of state (EOS) parametrer $\left(\omega_{\mathrm{eff}}\right.$ being the effective equation of state).
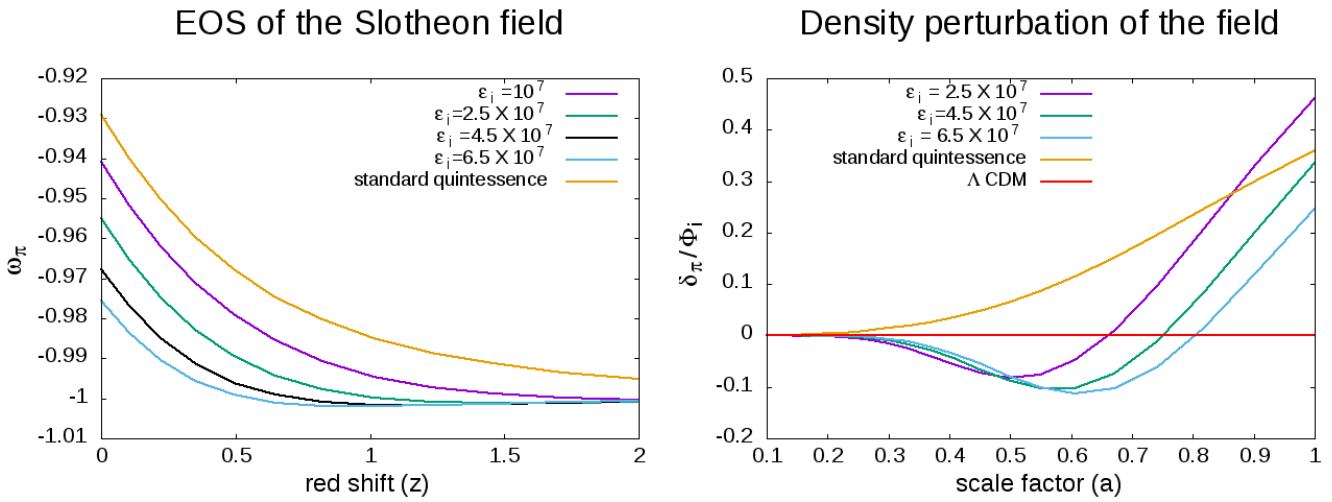

Figure 1: (a) Variations of Slotheon Dark Energy equation of state parameters (b) Evolutions of Slotheon field density fluctuations.

In Fig. 1(a) the variations of $\omega_{\pi}$ with $z$ are shown while in Fig. 1(b) we plot the evolutions of perturbations of Slotheon field $\delta_{\pi}$ with $a$. The Slotheon field EOS is different from standard quintessence for certain chosen initial values $\varepsilon_{i}$ of the parameter $\varepsilon$ but it tends to $\Lambda \mathrm{CDM}$ for higher $\varepsilon_{i}$. It may be noted here that higher values of $\varepsilon_{i}$ indicate greater affinity towards the Slotheon field Dark Energy. Similar trends are also observed for the evolution of perturbation for Slotheon field $\pi$ from Fig. 1(b).
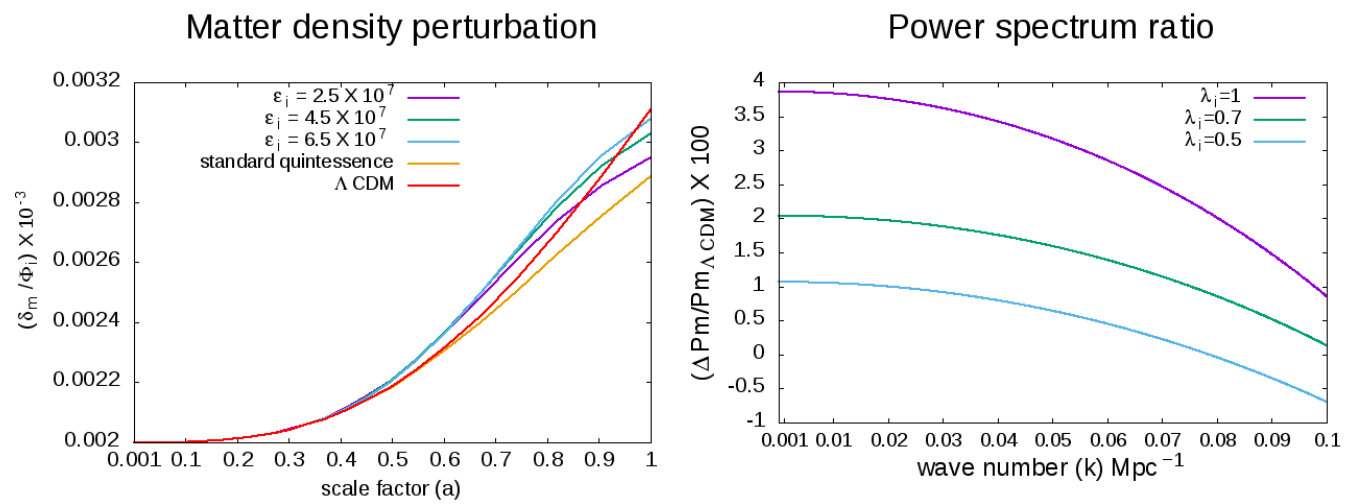

Figure 2: (a) Evolutions of matter density perturbationss considering the Slotheon field. (b) Matter power spectrum in presence of Slotheon field. Shown is the difference (in \%) of the power spectra for the $\Lambda \mathrm{CDM}$ model and Slotheon field model and their variations.

In Fig. 2(a) we show the variations of matter perturbations $\delta_{m}$ with the scale factor $a$. Although initially the $\delta_{m}$ calculated with Slotheon field coincides with those obtained considering $\Lambda$ CDM and standard quintessence models, the former (Slotheon) deviates from both the latter models with the advent of Dark Energy domination, as $a$ increases.

We also compute the matter power spectrum for the Slotheon field and compare it with that of 
$\Lambda \mathrm{CDM}$. The matter power spectrum is defined in terms of matter density fluctuations $\delta_{m}$ as

$$
P m=\left\langle\left|\delta_{m}(k, a)\right|^{2}\right\rangle .
$$

In order to compare $P m$ for Slotheon field with that of $\Lambda$ CDM model we define a quantity $X$ as

$$
X=\frac{P m_{\Lambda \mathrm{CDM}}-P m_{\text {slotheon }}}{P m_{\Lambda \mathrm{CDM}}} \times 100=\frac{\Delta P m}{P m_{\Lambda \mathrm{CDM}}} \times 100 .
$$

In Fig. 2(b) we plot the variations of $X$ with wave number $k$ for three different initial values $\lambda_{i}$ of the parameter $\lambda$. It appears from Fig. 2(b) that the percentage difference is more for smaller wave number but it reduces as the value of $k$ increases. Also $X$ decreases with the decrease of $\lambda_{i}$. This can be explained from the fact that as $\lambda_{i}$ decreases the potential $V(\pi)$ tends to be more flat and thereby approaches $\Lambda \mathrm{CDM}$ model.

\section{Summary}

Within the framework of Slotheon Dark Energy model (inspired by Dvali, Gabadadze and Porrati (DGP) model related to extra dimension) we explore the Dark Energy perturbations and growths with redshift and compare them with those for $\Lambda \mathrm{CDM}$ model and standard quintessence model. We have also computed the matter power spectrum for Slotheon scalar field model and comparisons have been made with the power spectrum when $\Lambda \mathrm{CDM}$ model is considered. we find that in certain circumstances the Slotheon field model is more akin to the $\Lambda \mathrm{CDM}$ model results. Similar trend is also observed for the case of power spectrum.

\section{References}

[1] P.J. Steinhardt, L.M. Wang and I. Zlatev, Phys. Rev. D 59, 123504 (1999).

[2] S. Tsujikawa, Class. Quant. Grav. 30, 214003 (2013).

[3] G. Dvali, G. Gabadadze, and M. Porrati, Phys. Lett. B 485, 208 (2000).

[4] M.A. Luty, M. Porrati, and R. Rattazzi, JHEP 0309, 029 (2003).

[5] B. Jain and J. Khoury, Ann. Phys. 325, 1479 (2010).

[6] D. Adak, A. Ali and D. Majumdar, Phys. Rev. D 88, 024007 (2013).

[7] S. Dodelson, Modern cosmology, Academic press, San diego, California (2003).

[8] L. Amendola and S. Tsujikawa, Theory and Observations of Dark Energy, Cambridge University press, New York, (2010). 